\title{
Improving the use of sputum cultures in lower respiratory tract infection
}

\author{
${ }^{1}$ OL Moncayo-Nieto, ${ }^{2} \mathrm{P}$ Reid, ${ }^{3} \mathrm{IF}$ Laurenson, ${ }^{4} \mathrm{AJ}$ Simpson \\ ${ }^{1}$ Clinical Lecturer in Medical Microbiology, University of Edinburgh, UK; ${ }^{2}$ ST6 in Respiratory Medicine, Royal Infirmary of Edinburgh, UK; \\ ${ }^{3}$ Consultant, Department of Clinical Microbiology, Royal Infirmary of Edinburgh, UK; ${ }^{4}$ Professor of Respiratory Medicine, Institute of Cellular \\ Medicine, Medical School, Newcastle University, Newcastle upon Tyne, UK
}

\section{ABSTRACT}

Background: The clinical value of sputum culture in suspected lower respiratory tract infection (LRTI) remains contentious. The quality of samples submitted significantly impacts their clinical usefulness.

Methods: Using pre-defined criteria we prospectively analysed the appropriateness of sputum samples submitted from consecutive patients with suspected LRTI attending two acute hospital units over ten weeks. We then provided an education package for staff on when and how to collect appropriate sputum samples, and repeated the evaluation.

Results: Our intervention reduced sample numbers from 347 to 133 , simultaneously increasing the proportion of appropriately sent samples from 40.5 to $60.2 \%(p=0.00 \mathrm{I})$ and reducing cost. Appropriate sampling was associated with a higher yield of pathogens (relative risk I.5I, 95\% confidence intervals I.03-2.2I, $\mathrm{p}=0.03$ ). The rate at which sputum samples appeared to alter clinicians' management remained low and constant at $18 \%$ pre- and post-intervention.

Conclusion: A simple educational intervention can significantly increase appropriateness of sputum sampling, reducing workload and cost.

KEYWORDS: Sputum culture, lower respiratory tract infection

DECLARATION OF INTERESTS No conflicts of interest declared.
Correspondence to J Simpson, Institute of Cellular Medicine, 4th Floor William Leech Building, Medical School, Newcastle University, Framlington Place, Newcastle upon Tyne NE2 4HH

tel. $+44(0) 1912227770$ e-mail j.simpson@ncl.ac.uk

\section{INTRODUCTION}

The role of sputum sampling in the management of lower respiratory tract infection (LRTI) has been controversial for some time. Evidence suggests that sputum sampling has low sensitivity, specificity and impact on treatment decisions. ${ }^{1-4}$ Ideally antibiotic treatment should be tailored to the causative pathogen but at presentation the aetiology is rarely known, and patients therefore commonly receive empirical, often broad-spectrum antibiotics.

In theory, a pathogen-directed approach to treatment has many advantages such as narrowing the therapeutic index of antibiotics, thus reducing adverse events and the risk of antimicrobial resistance. In addition, it allows the identification of unusual or unexpected pathogens, antibiotic-resistant organisms or those of epidemiological or public health significance. In practice the diagnostic usefulness of sputum cultures is limited by use of prior antibiotics, ${ }^{5}$ or the patient's inability to produce sputum. Generally, when sputum is obtained, the proportion of 'high quality' samples ranges from $25-60 \%$, though higher rates have been reported..$^{6-11}$ The variable yield in sputum cultures is also influenced by the transport, processing and interpretation of samples.

With the resultant low diagnostic yield and minimal impact on therapeutic decisions, routine sputum cultures have been discouraged. The Infectious Diseases Society of America/American Thoracic Society guidelines recommend that sampling in hospitalised adult patients be restricted to those able to provide a high quality pretreatment specimen and where quality performance measures for sputum collection, transport and processing of samples can be met, particularly in patients with severe community-acquired pneumonia (CAP). ${ }^{12}$ The British Thoracic Society similarly recommend that in hospitalised adults, samples should only be sent from patients able to expectorate purulent samples, who have not received prior antibiotics, or who have failed to respond to initial therapy, or have severe CAP. ${ }^{13}$

On the other hand, in an era of increasing antibiotic resistance, foreign travel and overuse of antibiotics, there is a need to improve the performance of diagnostic 
tests. High quality, informative and diagnostically useful sputum sampling is non-invasive, and should be achievable with relatively little staff training.

With this background in mind the main objective of this study was to evaluate the practice of sputum sampling, then to determine whether a simple education programme could impact upon the quality and cost of sampling.

\section{MATERIALS AND METHODS}

\section{Study design}

A prospective observational study was performed over ten weeks during the winter. Data were collected on consecutive sputum samples sent for microbiological analysis from the Acute Medical Assessment and Respiratory Units of an 865-bed teaching hospital. On the basis of the data collected, an educational package was distributed to staff in the two units concerned. The study was then repeated, using an identical format, in the same two units over ten weeks of a subsequent winter period.

\section{Data collection and definitions used in the study}

Patient demographics, prior use of antibiotics, current treatment and drug charts were monitored until the patient was discharged from hospital or death. Times of sample production, delivery to the laboratory and the issuing of microbiology reports were recorded. Samples were excluded from analysis if patient information was unavailable. Samples requesting analysis solely for mycobacteria were also excluded.

The microbiological quality of sputum specimens was recorded (based on macroscopic appearances) as salivary, mucoid, mucopurulent or purulent by trained staff in the Department of Clinical Microbiology. In our institution routine Gram staining of sputum samples is not performed.

The following definitions were determined prior to the commencement of the study:

- Working diagnosis: the diagnosis made by the first senior physician to assess the patient clinically.

- Appropriate sample: any sample taken prior to the start of antibiotics or where failure to improve despite appropriate antibiotic treatment was documented.

- Inappropriate sample: any sample taken after the initiation of antibiotic treatment; any sample received in the laboratory more than 48 hours after expectoration; samples sent within three days of an appropriate sample being submitted; and samples considered to consist of saliva according to laboratory staff.
- Positive culture: the isolation of a respiratory tract pathogen.

- Negative culture: no growth; growth consistent with upper respiratory tract flora; or growth of coliforms and/or yeasts in patients already receiving antibiotics.

\section{Educational material issued as a result of the first} round of sampling

A single-page document was prepared, summarising the rate of inappropriate sputum sampling and outlining criteria for appropriate sampling. The sheet was distributed to each nursing bay and each doctors' room in the two clinical areas involved. The purpose of the summary was discussed with staff at the time of distribution and reinforced at a hospital 'grand round'. Staff were asked to display the summary sheets in a prominent place within their working area, and were informed that the effectiveness of sampling would be recorded in the future at an unspecified time. The second round of the observational study was performed four weeks after distribution of the educational material.

Cost analyses for both periods of collection were performed using the prices of sputum samples in our hospital at the end of the second round of sampling. The overall cost of processing a sputum sample was $£ \mid 6.03$.

\section{Statistics}

Descriptive data are presented as means +/- standard deviation (SD) or range, where appropriate. Categorical variables were compared using the chi-squared test or Fisher's exact test, where appropriate. The level of significance was set at $p<0.05$. All statistical values were calculated using a statistical software package.

\section{RESULTS}

In the pre-intervention study, 417 consecutive sputum samples from 263 patients were sent for microbiological analysis. A total of 44 samples from 33 patients were excluded because of incomplete or missing clinical records; 26 samples were processed solely for mycobacteria and were excluded from analysis. Final analysis therefore involved 347 sputum samples from 221 patients.

In the post-intervention study, 169 consecutive sputum samples were submitted, of which 12 were excluded due to incomplete clinical data; 24 samples processed solely for mycobacteria were excluded. Final analysis therefore involved I 33 samples from 121 patients.

The total numbers of patients managed in the relevant units during the study periods were 3,642 for the preintervention and 3,459 for the post-intervention study. The number of samples submitted per patient was 
significantly lower in the post-intervention period, odds ratio: $1.78,95 \%$ confidence interval $(\mathrm{Cl})$ : I.42$2.24(p<0.000 \mathrm{I})$. The total cost of submitted samples was $£ 5,562.41$ in the pre-intervention period and $£ 2,131.99$ in the post-intervention period. The absolute saving equated to $£ 3,430.42$, approximating to $£ 17,800$ per annum.

The two study groups were similar with regard to age, sex distribution and the proportion of samples derived from each hospital unit (Table I). The range of working diagnoses in the two study periods was also similar, though exacerbations of asthma were diagnosed more frequently in the post-intervention period (Table 2).

TABLE I Patient demographics
\begin{tabular}{|l|c|c|}
\hline & $\begin{array}{c}\text { Pre- } \\
\text { intervention } \\
\text { Cycle I }\end{array}$ & $\begin{array}{c}\text { Post- } \\
\text { intervention } \\
\text { Cycle 2 }\end{array}$ \\
\hline Weeks & 10 & 10 \\
\hline Time period & Nov - Jan & Feb - May \\
\hline Sputum samples (n) & 347 & 133 \\
\hline Patients (n) & 221 & 121 \\
\hline $\begin{array}{l}\text { Female/male ratio } \\
\text { (\% female) }\end{array}$ & $121 / 100(54.8)$ & $63 / 58(52.1)$ \\
\hline Age in years (range) & $67(14-91)$ & $63(14-94)$ \\
\hline $\begin{array}{l}\text { Origin of samples by } \\
\text { unit }\end{array}$ & & \\
$\begin{array}{l}\text { Acute Medical } \\
\text { Assessment (\%) }\end{array}$ & $\begin{array}{l}129(37.2) \\
\text { Respiratory (\%) }\end{array}$ & $\begin{array}{c}45(33.8) \\
88(66.2)\end{array}$ \\
\hline $\begin{array}{l}\text { Data are presented as total numbers and mean (range) } \\
\text { where indicated. }\end{array}$
\end{tabular}

The proportion of samples yielding a positive result was similar in both study periods (Table 3). The frequency of isolation of individual pathogens was generally conserved in the two study periods. However Streptococcus pneumoniae was significantly more likely to be isolated in the first study period and Pseudomonas aeruginosa in the second. There was a trend towards more frequent isolation of Moraxella catarrhalis in the first period. No sputum sample grew more than one pathogen in the post-intervention period, whereas 20 samples grew two pathogens in the pre-intervention period. Nine samples were positive for Mycobacterium tuberculosis in the preintervention period (Table 3). These were derived from two patients, who submitted four and five positive samples, respectively. No samples were positive for $M$. tuberculosis in the post-intervention period.

The appropriateness of sputum sampling and the influence upon clinical decision-making is illustrated in Figure I. In the pre-intervention study $39.5 \%$ of samples were considered appropriate whereas $60 \%$ were considered appropriate in the post-intervention study, $\mathrm{p}=0.00 \mathrm{I}$.
TABLE 2 Working diagnoses

\begin{tabular}{|c|c|c|c|}
\hline Diagnosis & $\begin{array}{c}\text { Pre- } \\
\text { intervention } \\
\text { Cycle I } \\
n=221\end{array}$ & $\begin{array}{c}\text { Post- } \\
\text { intervention } \\
\text { Cycle } 2 \\
n=|2|\end{array}$ & p value \\
\hline $\begin{array}{l}\text { Exacerbation } \\
\text { of chronic } \\
\text { obstructive } \\
\text { pulmonary } \\
\text { disease } \\
\text { (COPD) }\end{array}$ & $86(38.9)$ & 35 (28.9) & 0.07 \\
\hline Pneumonia & $59(26.7)$ & $26(21.5)$ & 0.29 \\
\hline $\begin{array}{l}\text { Lower } \\
\text { respiratory } \\
\text { tract infection } \\
\text { (LRTI) } \\
\text { without } \\
\text { consolidation }\end{array}$ & $35(15.8)$ & $24(19.8)$ & 0.37 \\
\hline Bronchiectasis & $8(3.6)$ & $7(5.8)$ & 0.41 \\
\hline Lung cancer & 7 (3.2) & $3(2.5)$ & 1.00 \\
\hline $\begin{array}{l}\text { Exacerbation } \\
\text { of asthma }\end{array}$ & $4(1.8)$ & $12(9.9)$ & 0.001 \\
\hline Miscellaneous & $22(10.0)$ & $14(11.6)$ & 0.71 \\
\hline
\end{tabular}

TABLE 3 Sputum culture yield

\begin{tabular}{|c|c|c|c|}
\hline & $\begin{array}{c}\text { Pre- } \\
\text { intervention } \\
\text { Cycle I } \\
n=347\end{array}$ & $\begin{array}{c}\text { Post- } \\
\text { intervention } \\
\text { Cycle } 2 \\
n=133\end{array}$ & $\begin{array}{c}\mathbf{p} \\
\text { value }\end{array}$ \\
\hline Negative culture & $230(66.3)$ & $92(69.2)$ & \multirow[t]{2}{*}{0.58} \\
\hline Positive culture & 117 (33.7) & $41(30.8)$ & \\
\hline $\begin{array}{l}\text { Haemophilus } \\
\text { influenzae }\end{array}$ & 31 (8.9) & II (8.3) & 1.00 \\
\hline $\begin{array}{l}\text { Methicillin- } \\
\text { resistant S. } \\
\text { aureus (MRSA) }\end{array}$ & $19(5.5)$ & $9(6.8)$ & 0.47 \\
\hline $\begin{array}{l}\text { Methicillin- } \\
\text { sensitive S. } \\
\text { aureus (MSSA) }\end{array}$ & $6(1.7)$ & $3(2.3)$ & 0.69 \\
\hline $\begin{array}{l}\text { Pseudomonas } \\
\text { aeruginosa }\end{array}$ & $8(2.3)$ & $10(7.5)$ & 0.0037 \\
\hline $\begin{array}{l}\text { Streptococcus } \\
\text { pneumoniae }\end{array}$ & $17(4.9)$ & I $(0.8)$ & 0.044 \\
\hline $\begin{array}{l}\text { Mycobacterium } \\
\text { tuberculosis }\end{array}$ & $9(2.6)$ & 0 & 0.11 \\
\hline $\begin{array}{l}\text { Moraxella } \\
\text { catarrhalis }\end{array}$ & $21(6.1)$ & $2(1.5)$ & 0.067 \\
\hline Miscellaneous & $26(7.5)$ & $5(3.8)$ & 0.15 \\
\hline
\end{tabular}

Data are presented as absolute numbers with percentage in parenthesis. Twenty samples in cycle I yielded >I pathogen, i.e. 117 samples yielded a positive culture, and 137 pathogens were isolated from these samples. In calculating percentages the denominator was taken to be II7. During cycle two none of the samples grew a second organism. 


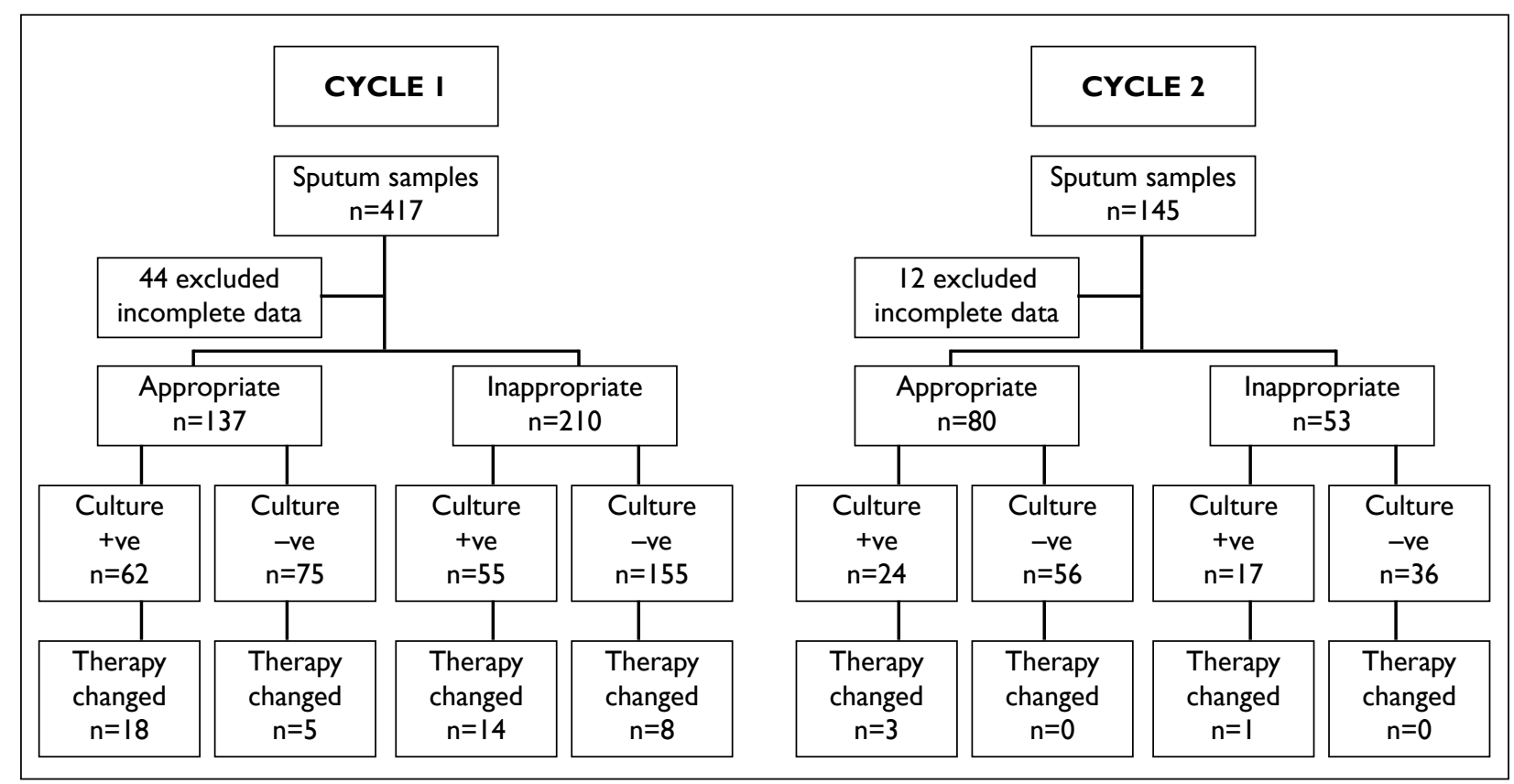

FIGURE I Appropriateness of sputum sampling, microbiological yield, and clinical decision-making before and after intervention. The panel on the left describes details relating to sputum sampling before intervention and the panel on the right describes sampling after intervention.

In the pre-intervention study, submission of an appropriate sample was more likely to yield a positive culture ( $45 \%$ of samples) than an inappropriate sample $(26 \%)(p<0.00 I)$. In contrast, the post-intervention study was characterised by an equal proportion of positive cultures among samples considered to be appropriate $(30 \%)$ or inappropriate $(32 \%)(p=0.84)$. When considering both cycles together, the relative risk of producing a positive result from an appropriate sample was I.5I (95\% Cl: 1.03 to $2.21 ; p=0.03$ I 2). In contrast, the relative risk of producing a positive culture result from an inappropriate sample was 0.82 ( $95 \% \mathrm{Cl} 0.52$ to 1.28$)$.

In the pre-intervention study the principal reason for inappropriate samples was collection after initiation of antibiotics (84.7\%), followed by duplicate samples (1 I.9\%), salivary samples (1.9\%) and samples that arrived in the laboratory beyond 48 hours ( $1.5 \%$ ). In the post-intervention study the corresponding reasons were samples taken after antibiotics were started (85\%) and duplicate samples (I5\%). No salivary samples were received.

In terms of patient management, in the pre-intervention study 23 appropriate sputum samples (16.8\%) and 22 inappropriate sputum samples (10.5\%) appeared to direct a change in antibiotic prescription. In the postintervention study three appropriate samples (3.8\%) and one inappropriate sample (1.9\%) appeared to result in a change in antibiotic prescription.

The proportion of samples that a) were considered appropriate and b) grew a pathogen was 17.9\% (62 of 347 samples) in the pre-intervention period and $18.0 \%$ (24 of 133 samples) in the post-intervention period.

\section{DISCUSSION}

Our data suggest that in the absence of specific education, a high proportion of inappropriate sputum samples are sent to diagnostic laboratories, incurring costs that could be diverted to other health services. However a simple and inexpensive intervention led to a significant decline in the number of samples and an associated cost saving.

Furthermore, the proportion of samples considered 'appropriate' was significantly increased after the intervention. During both cycles of the study, 'appropriate' samples were significantly more likely to yield a positive culture result than 'inappropriate' samples, broadly in keeping with findings in patients with chronic obstructive pulmonary disease (COPD). ${ }^{14}$ Other studies have shown that the quality of sampling improves after an educational intervention similar to the one used in our study, ${ }^{6}$ and that diagnostic yield for S. pneumoniae can potentially increase substantially with improvement in sputum quality. ${ }^{15}$

In general our findings are supportive of literature showing that sputum samples have little overall impact upon practical management of patients..$^{2-4,16,17}$ Prior to our intervention approximately $13 \%$ of sputum samples (45/347) appeared to lead to a change in management, but half of these samples were 'inappropriate'. Even fewer samples (around 3\%, 4/I33) directly altered prescribing after the intervention.

It may however be useful to consider the relative importance of culture results in their specific context. 
For example, it could be argued that a sputum sample which is both appropriate and grows a pathogen is clinically useful whether antibiotics are changed or not, on the grounds that such samples can improve physicians' confidence to continue existing therapy. If this argument is accepted, the proportion of 'useful' samples remained constant at $18 \%$ pre- and postintervention, despite the significant reduction in samples submitted. Nevertheless, it is interesting to observe that the proportion of inappropriate samples due to collection after initiation of antibiotics remained constant during both study periods.

In contrast, it could be argued that alterations in management based on 'inappropriate' samples represent a clinical risk. In this regard, before intervention $10.5 \%$ of samples led to a change in antibiotics based on inappropriate samples, as compared to $1.9 \%$ postintervention. It therefore appears that our intervention reduced the number of inappropriate samples submitted, and was also associated with improved management outcomes. We cannot be absolutely certain however that positive cultures in inappropriate samples never represent pathogens.

Our results suggest reluctance among physicians to alter therapy based on a negative sputum culture. Patient management was influenced in only $4 \%$ of cases of negative cultures (13/322) when considering both cycles together. We do not have precise data regarding how many of these patients were given empirical antibiotics, but certainly over half had been prescribed antibiotics empirically. These data suggest that physicians remain reluctant to discontinue antibiotics once they are started, even when cultures are negative. The reasons around this are probably multi-factorial, one potentially being a lack of confidence in the quality of samples being sent. The implication however is that there is scope for a further reduction of inappropriate antibiotic prescription in patients with suspected LRTI.

While our results reveal some clear patterns, there were also some limitations in our study. Firstly, while our data point to a clear reduction in inappropriate sampling, we cannot exclude the possibility that in some cases samples that would have been appropriate and culture positive may potentially not have been submitted in the post-intervention period. While the post-intervention period was associated with a $75 \%$ reduction in inappropriate samples (from 210 to 53 ), the number of appropriate samples fell from 137 to 80 . The significance of this is unclear and we cannot make meaningful inferences from this study, as we have no data on outcomes in patients with possible LRTI who had no sputum sampling, nor do we know how many of this cohort received antibiotics in either study period. Our most informed opinion is that empirical antibiotics are relatively widely used in patients with possible infection but no sputum sample, potentially reducing the impact of 'missing' a few genuine positive samples in the postintervention period.

Secondly, this was a single-institution study, in which we only studied two units (those which we believed to be submitting sputum samples most frequently) over two time periods. Although we studied two winter periods of equal length, we cannot entirely exclude the possibility that the pool of pathogens in the community was different in each period. Our results (Figure I) suggest that $45 \%$ of appropriate samples were culture positive in the pre-intervention period, as compared with $30 \%$ in the post-intervention period, potentially implying exposure of the community to more (and/or more virulent) organisms in the former. While this phenomenon might potentially bias the absolute number of samples taken in favour of the intervention, it should not influence the ratio of appropriate to inappropriate samples. Furthermore, influenza activity was known to be at similarly low levels in both study periods, perhaps making it less likely that results were influenced by significant variations in exposure of the community to respiratory pathogens. Importantly, while our findings suggest that our intervention can reduce the proportion of inappropriate samples, and potentially reduce changes in management based on inappropriate samples, it would clearly take a much larger, more complex and longer study to prove that beneficial changes in appropriateness of sampling convert into improvements in hard clinical outcomes.

Third, our study was not designed to establish whether, or how rapidly, the beneficial effects of our intervention declined over time. One study has suggested that longterm changes in practice are harder to influence. ${ }^{6}$ Future studies should consider longer follow-up periods to determine the duration of the intervention effect, and whether reinforcement is required (and if so, how often). Fourth, we assessed changes in management based on prescription charts, and we have no data on why decisions to change treatment (or not) were made.

Finally, while we regard the decision to consider all sputum samples sent from the two units to be a strength (reflecting 'real life' clinical practice rather than focusing on any specific clinical diagnosis), we acknowledge that this approach brings its own constraints. For example, the exclusion of samples sent for mycobacterial analysis prevents the study from reflecting every patient with suspected LRTI. We excluded mycobacterial analysis specifically in order to avoid confounding our data with a distinct subgroup of patients who by definition would have multiple samples sent where possible. Furthermore, our study did not have the capacity to address the utility of sputum in diagnosing 'atypical' pathogens or viruses, which are increasingly recognised as contributing to LRTI. ${ }^{18,19}$ 
In summary, a simple educational intervention can be associated with a significant reduction in the number of inappropriate sputum samples sent to the laboratory, with a higher yield of positive cultures in appropriate samples, and possibly with improved management decisions based on 'inappropriate samples'. Further studies will be required to determine whether this approach is sustainable and whether it can influence clinical outcomes.

\section{REFERENCES}

I Lentino JR, Lucks DA. Nonvalue of sputum culture in the management of lower respiratory tract infections. J Clin Microbiol 1987; 25:758-62.

2 Woodhead MA, Arrowsmith J, Chamberlain-Webber $R$ et al. The value of routine microbial investigation in community-acquired pneumonia. Respir Med 1991; 85:3 13-7. http://dx.doi.org/I0.1016/ S0954-6 I I I (06)80 I03-4

3 Sanyal S, Smith PR, Saha AC et al. Initial microbiologic studies did not affect outcome in adults hospitalized with communityacquired pneumonia. Am J Respir Crit Care Med 1999; 160:346-8. http://dx.doi.org/I0.I I64/ajrccm. I60.1.9806048

4 Ewig S, Schlochtermeier M, Göke $\mathrm{N}$ et al. Applying sputum as a diagnostic tool in pneumonia: limited yield, minimal impact on treatment decisions. Chest 2002; 121:|486-92. http://dx.doi. org/10.1378/chest.121.5.1486

5 Miyashita N, Shimizu H, Ouchi $K$ et al. Assessment of the usefulness of sputum Gram stain and culture for diagnosis of community-acquired pneumonia requiring hospitalization. Med $\mathrm{Sci}$ Monit 2008; I4:CR I7I-6.

6 Jacobson JT, Burke JP, Jacobson JA. Ordering patterns, collection, transport, and screening of sputum cultures in a community hospital: evaluation of methods to improve results. Infect Control I981; 2:307-II.

7 Rosón B, Carratalà J, Verdaguer R et al. Prospective study of the usefulness of sputum Gram stain in the initial approach to community-acquired pneumonia requiring hospitalization. Clin Infect Dis 2000; 31:869-74. http://dx.doi.org/10.1086/3/8I5I

8 García-Vázquez E, Marcos MA, Mensa J et al. Assessment of the usefulness of sputum culture for diagnosis of community-acquired pneumonia using the PORT predictive scoring system. Arch Intern Med 2004; I64: I807-I I.http://dx.doi.org//0.100 I/archinte. 164.16.1807

9 Roche N, Kouassi B, Rabbat A et al.Yield of sputum microbiological examination in patients hospitalized for exacerbations of chronic obstructive pulmonary disease with purulent sputum. Respiration 2007; 74: 19-25. http://dx.doi.org/I0.1 I59/000093/58

10 Larsen MV, Janner JH, Nielsen SD et al. Bacteriology in acute exacerbation of chronic obstructive pulmonary disease in patients admitted to hospital. Scand J Infect Dis 2009; 41:26-32. http://dx. doi.org// 0.1080/00365540802484828

\section{Acknowledgements}

We are very grateful to Thomas Hamilton, Sander Lipka, Morag Sutherland and Kenneth Muir for their help in preparing this work.

II Shariatzadeh MR, Marrie TJ. Does sputum culture affect the management and/or outcome of community-acquired pneumonia? East Mediterr Health J 2009; I 5:792-9.

I2 Mandell LA, Wunderink RG, Anzueto A et al. Infectious Diseases Society of America/American Thoracic Society consensus guidelines on the management of community-acquired pneumonia in adults. Clin Infect Dis 2007; 44:S27-S72. http://dx.doi. org/10.1086/5III59

I3 Lim WS, Baudouin SV, George SC et al. BTS Guidelines for the management of community-acquired pneumonia in adults: update 2009. Thorax 2009; 64 (Suppl 3):I-55. http://dx.doi.org//0.1 |36/ thx.2009.121434

I4 Telgen MC, Brusse-Keizer MG, van der Valk PD et al. Impact on clinical decision making of quality control standards applied to sputum analysis in COPD. Respir Med 20I I; 105:37I-6. http://dx. doi.org/10.1016/j.rmed.2010.10.009

I5 Musher DM, Montoya R, Wanahita A. Diagnostic value of microscopic examination of Gram-stained sputum and sputum cultures in patients with bacteremic pneumococcal pneumonia. Clin Infect Dis 2004; 39:165-9. http://dx.doi.org/10.1086/42I497

16 Theerthakarai R, El-Halees W, Ismail M et al. Nonvalue of the initial microbiological studies in the management of nonsevere community-acquired pneumonia. Chest 2001; II9:181-4. http:// dx.doi.org/10.1378/chest. I19.1.181

17 Lidman C, Burman LG, Lagergren A et al. Limited value of routine microbiological diagnosis in patients hospitalized for communityacquired pneumonia. Scand J Infect Dis 2002; 34:873-9. http:// dx.doi.org// 0.1080/003655402/000026967

I8 Ruiz M, Ewig S, Marcos MA et al. Etiology of community-acquired pneumonia: impact of age, comorbidity and severity. Am J Respir Crit Care Med 1999; 160:397-405. http://dx.doi.org/I0.II64/ ajrccm. 160.2.9808045

19 Díaz A, Barria P, Niederman $M$ et al. Etiology of communityacquired pneumonia in hospitalized patients in Chile: the increasing prevalence of respiratory viruses among classic pathogens. Chest 2007; | 3 |:779-87. http://dx.doi.org/ |0.|378/chest.06-1800 